\title{
Effects of dust particle internal structure on light scattering
}

\author{
O. Kemppinen ${ }^{1,2}$, T. Nousiainen ${ }^{1}$, and G. Y. Jeong ${ }^{3}$ \\ ${ }^{1}$ Earth Observation, Finnish Meteorological Institute, P.O. Box 503, 00101, Helsinki, Finland \\ ${ }^{2}$ Department of Applied Physics, Aalto University, Espoo, Finland \\ ${ }^{3}$ Department of Earth and Environmental Sciences, Andong National University, Andong 760-749, Republic of Korea
}

Correspondence to: O. Kemppinen (osku.kemppinen@fmi.fi)

Received: 8 June 2015 - Published in Atmos. Chem. Phys. Discuss.: 28 July 2015

Revised: 8 October 2015 - Accepted: 19 October 2015 - Published: 28 October 2015

\begin{abstract}
There is a large variety of internal structures inside atmospheric dust particles, making them inherently inhomogeneous. Such structures may have a large effect on ground-level and atmospheric radiation. So far, dust particle internal structures and their effect on the light scattering properties have proved to be hard to quantify, in part due to challenges in obtaining information about these structures. Recently, internal structures of individual dust particles were revealed through focused ion beam milling and analyzed. Here, we perform a sensitivity study to evaluate the optical impacts of some of the typical internal structures revealed. To obtain suitable model particles, the first step is to generate inhomogeneous particles with varying internal structures by using an algorithm that is based on three-dimensional Voronoi tessellation. The parameters for the particle generation are obtained from studies of real-world Asian dust particles. The second step is to generate homogeneous versions of the generated particles by using an effective-medium approximation, for comparison. Third, light scattering by both versions of these particles is simulated with discrete dipole approximation code. This allows us to see how different internal structures affect light scattering, and how important it is to account for these structures explicitly. Further, this allows us to estimate the potential inaccuracies caused by using only homogeneous model particles for atmospheric studies and remote-sensing measurements. The results show that the effects vary greatly between different kinds of internal structures and single-scattering quantity considered, but for most structure types the effects are overall notable. Most significantly, hematite inclusions in particles impact light scattering heavily. Furthermore, internal pores and hematite-rich coating both affect some form of light scattering noticeably.
\end{abstract}

Based on this work, it seems that it is exceedingly important that the effects of dust particle internal structures on light scattering are accounted for in a wide variety of applications.

\section{Introduction}

Mineral dust particles are an important part of the atmosphere (Zender et al., 2003). Their impact on incoming ground-level solar radiation, including aerosol radiative forcing, can be considerable (Durant et al., 2009; Haywood et al., 2011a, b; Osborne et al., 2011). At areas with high dust concentrations, their effect on the amount of incoming radiative energy at the surface can be roughly ten percent when the Sun is at the zenith, and even more when it is close to the horizon. Interestingly, this effect can either increase or decrease the amount of radiative energy reaching the surface (Forster et al., 2007). Due to its abundance and impact, accounting for dust is imperative in most radiative balance calculations and atmospheric remote sensing. However, due to the large variance in dust effects on radiation, the impacts are challenging to account for, particularly when using simplified models.

The reasons for the large variance of the impact of dust on radiation can be many, but in addition to the obvious variations in particle size and concentration, shapes, surface roughness characteristics and internal structures may play a role. It is known that many dust particles contain materials with significant dielectric contrast, for example iron oxides or internal pores. Transmission electron microscopic (TEM) analysis of cross-sections of single particles showed that internal pores of varying sizes are common features of 
Asian dust particles. Additionally, submicron iron oxides, such as hematite and goethite, are often distributed within clay medium (Jeong and Nousiainen, 2014). Furthermore, it has been shown by, e.g., Vilaplana et al. (2006), Nousiainen et al. (2011a, b, 2003), and Muinonen et al. (2009) that inhomogeneity can affect light scattering by particles significantly, and that scattering by such particles is not easily mimicked by ensembles of simple homogeneous model particles.

While particle size distributions and many other population-level parameters are possible to measure by remote sensing (Chou et al., 2008; Kim et al., 2008), shapes and internal structures are much harder to determine. For example, derivation of the true three-dimensional shape requires application of atomic force microscopy (e.g. Chou et al., 2008; Woodward et al., 2015), or stereogrammetry from electron microscope images (Lindqvist et al., 2014). Internal structures can be analyzed, e.g., by slicing open dust particles with a focused ion beam, as done by Jeong and Nousiainen (2014). The impacts of real shapes and internal structures on scattering have barely been touched, because it is very hard to retrieve the three-dimensional composition, and especially to measure light scattering by the same particles whose structures are retrieved. Without coincident data, the connection between optical properties and physical properties cannot be established.

Here we carry out a sensitivity study on the impacts of internal structures. Our approach is to create an algorithm that allows us to generate discrete dipole approximation (DDA) models of particles with desired types and amounts of internal structures, for which accurate light scattering simulations can be then easily run, thus allowing taking internal structures into account explicitly and accurately. What we aim to do is to generate a set of particles that are complex-shaped and irregular, as are real dust particles, and possess internal structure characteristics that resemble those observed in real dust. The clear benefit of this pure modeling approach is that it allows us to calculate accurate values for individual optical properties, linked to known individual physical properties. We will be testing how various internal structures change light scattering compared to a baseline version, which is composed only of optically similar minerals. Further, knowing the composition of the inhomogeneous particles completely allows us to calculate homogeneous versions of the same particles with an effective-medium approximation (EMA), and simulate light scattering by both the inhomogeneous and the homogeneous versions of the particles. This, then, allows us to quantify the errors in light scattering caused by using the homogeneous version of the particle instead of the true form of the particle.

The main purpose of this study is to investigate the effects of different types of internal structures on the singlescattering properties. We will be examining particles with various internal structures, such as empty cavities and materials with high real and imaginary refractive index, such as hematite and other iron oxides. The article is structured as follows: Sect. 2 introduces the physical dust particles on which the computational particle generator is based on, Sect. 3 summarizes the relevant single-scattering theory, Sect. 4 explains the model particle generator algorithm in detail, Sect. 5 shows the results of the scattering calculations for inhomogeneous and homogeneous particles and, finally, Sect. 6 contains the summary of the work, and discussion of the significance of the results.

\section{Internal structures in Asian dust particles}

Asian dust is an important mineral dust lifted from arid regions in northwestern China and southern Mongolia, and transported long range across East Asia and the North Pacific (Jeong et al., 2014, and references therein). The details of the particle interiors were recently discovered by Jeong and Nousiainen (2014). Most of the dust particles are composites of several mineral types of varying grain sizes. Relatively coarser minerals are quartz, plagioclase, K-feldspar, calcite, and muscovite, while finer minerals are illite-smectite series clay minerals and iron oxides. The basic internal structures of dust particles result from the agglomeration of these mineral grains in the source soils. Within the dust particles, coarser minerals tend to form separated grains, while very fine grains of clay minerals and iron oxides form tight aggregates. In clay-rich dust particles, coarser grains such as quartz, plagioclase and muscovite are enclosed in the clay medium, while in clay-poor particles, quartz and plagioclase grains are commonly crusted with coatings of clay agglomerates. Submicron irregular pores are common in the dust particles. Example internal structure and mineral distribution of an Asian dust particle $(3.8 \mu \mathrm{m}$ in diameter, Fig. 1a) is presented in Fig. 1b, showing a porous agglomerate of several mineral types. These particles were collected directly from air on a borosilicate glass-fiber filter using a total suspended particulate (TSP) sampler (Jeong and Nousiainen, 2014).

Jeong and Nousiainen (2014) grouped internal structures into single and polycrystalline cores of quartz, feldspars, calcite, and amphibole often with oriented clay coatings; clay agglomerates showing partially preferred orientations; and platy coarse phyllosilicates. Iron oxides in Asian dust are mostly goethite and hematite. Although their contents are low in mineral dust, their potential impact to optical properties is known to be significant (Sokolik and Toon, 1999; Lafon et al., 2006). Although they occur as their own agglomerates, they are commonly distributed as submicron grains through the clay medium as shown in Fig. 1c-d. Certainly, there must be wide ranges of internal structures and mineral compositions from porous to nonporous, coated to non-coated, preferred to randomly oriented, from monocrystalline to polycrystalline, and iron-poor to iron-rich (Jeong and Nousiainen, 2014). Currently, the statistics of structural types are not available yet because the number of dust particles subjected to TEM analysis is not sufficient. Evidently, 

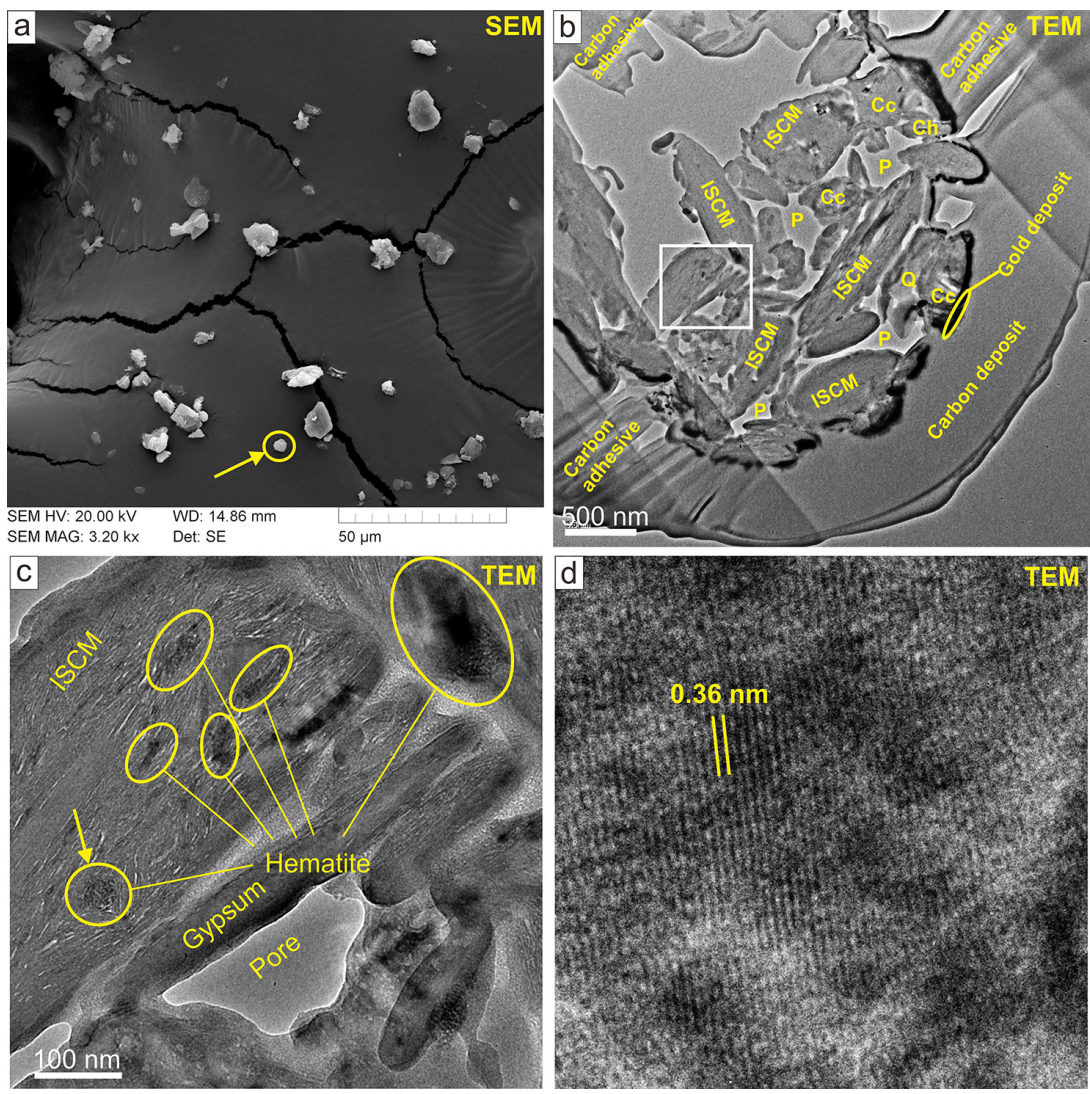

Figure 1. Example internal structure and mineral distribution in Asian dust particle. Panel (a) is an SEM image showing a dust particle of $3.8 \mu \mathrm{m}$ in diameter (arrow) processed with focused ion beam to prepare a thin slice for TEM analysis. Panel (b) is a TEM image of the slice showing mineral grains and interstitial pores. $\mathrm{Cc}=$ calcite, $\mathrm{Ch}=$ chlorite, $\mathrm{ISCM}=$ illite-smectite series clay minerals, $\mathrm{P}=$ pore, $\mathrm{Q}=$ quartz. Gold deposition was applied for electrical conduction for SEM observation. Carbon was deposited before focused ion beam process. Panel (c) is a magnified image from the square in Panel (b) showing the submicron hematite grains enclosed in the illite-smectite series clay minerals. Panel (d) is a lattice fringe image of hematite indicated as arrow in Panel (c).

further extensive TEM works in combination with highresolution scanning electron microscopy (SEM) are required to discover and characterize the various internal structures.

\section{Single-scattering concepts}

The foundation of all radiative effects comes from singlescattering interactions. The scattering matrix for a particle,

$$
\left(\begin{array}{c}
I_{\mathrm{s}} \\
Q_{\mathrm{s}} \\
U_{\mathrm{s}} \\
V_{\mathrm{s}}
\end{array}\right)=\frac{1}{k^{2} d^{2}}\left[\begin{array}{llll}
S_{11} & S_{12} & S_{13} & S_{14} \\
S_{21} & S_{22} & S_{23} & S_{24} \\
S_{31} & S_{32} & S_{33} & S_{34} \\
S_{41} & S_{42} & S_{43} & S_{44}
\end{array}\right]\left(\begin{array}{c}
I_{\mathrm{i}} \\
Q_{\mathrm{i}} \\
U_{\mathrm{i}} \\
V_{\mathrm{i}}
\end{array}\right),
$$

characterizes the single-scattering event by linking the properties of incident (i) and scattered (s) radiation by the particle. Here $[I, Q, U, V]^{\top}$ is the Stokes vector describing the properties of light: $I$ describes the intensity, $Q$ and $U$ the linear polarization, and $V$ the circular polarization of the wave. The wavenumber $k=2 \pi \lambda^{-1}$ is related to the radiation wavelength $\lambda$, and $d$ is the distance from the scattering particle.
Although the full matrix contains 16 elements, they are not independent, and under certain conditions, the matrix simplifies to only 6 independent elements: $S_{11}, S_{12}, S_{22}, S_{33}$, $S_{34}$, and $S_{44}$ (Hovenier and van der Mee, 2000). These conditions are that the particles be randomly oriented, and that either the particles are mirror symmetric, or the particles and their mirror particles are present in equal numbers. However, it has been shown by, e.g., Muñoz et al. (2012), Nousiainen and Kandler (2015) that even when these conditions are not strictly true, for ensembles of complex particles, such as dust, the scattering matrix closely conforms to the simplified form.

Here, we study the effects of internal structures primarily in terms of individual scattering matrix elements, from which all other effects can be determined. We also link these effects to relevant radiative transfer and remote-sensing quantities by calculating four scalar scattering quantities, which are described below.

The single-scattering albedo, $\bar{\omega}$, describes the amount of energy being scattered in a single scattering event compared to that being absorbed. To be precise, it is the ratio of scatter- 


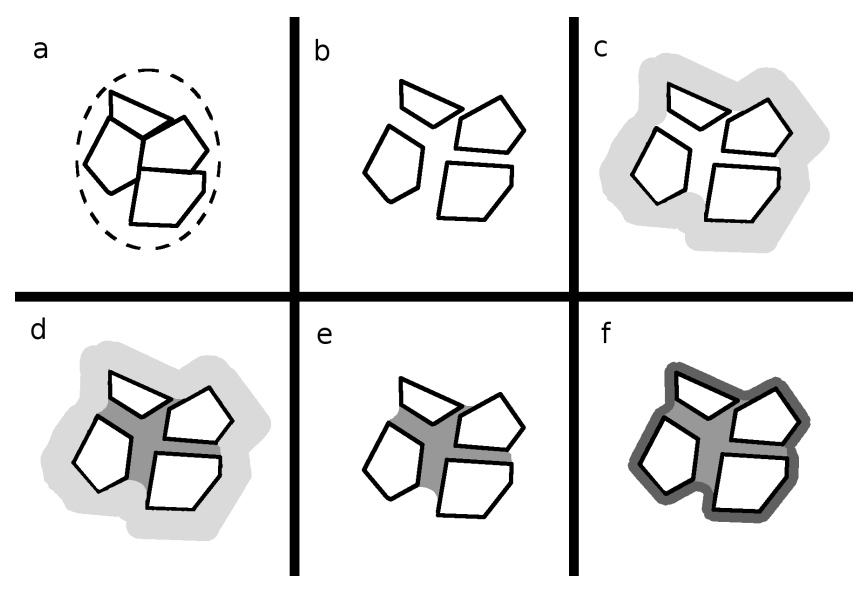

Figure 2. A schematic two-dimensional figure of the particle generation process. Panel (a) shows a simple four-cell tessellation, with the dashed ellipse showing an example culling surface. Panel (b) shows the cell separation. Panel (c) shows the formation of the concave hull, while Panel (d) shows the filling process based on the hull. Panel (e), shows the particle with no coating, and finally, Panel (f) shows the particle with coating added.

ing and extinction cross-sections:

$\bar{\omega}=\frac{C_{\text {sca }}}{C_{\text {ext }}}$

where $C_{\text {sca }}$ is the scattering cross-section, a measure of the total power scattered by the particle, and $C_{\text {ext }}$ is the sum of scattering and absorption cross-sections. In this work we use a derivative quantity, called the co-albedo, which is defined as $1-\bar{\omega}$.

The asymmetry parameter $g$ is used to describe how the scattered intensity varies between the forward $\left(\theta<90^{\circ}\right)$ and backward $\left(\theta>90^{\circ}\right)$ hemispheres of the scatterer, where $\theta$ is the scattering angle, i.e., the angular difference in propagation directions between the incident and the scattered radiation. The asymmetry parameter is obtained from $S_{11}$ by:

$g=\frac{2 \pi}{k^{2} C_{\text {sca }}} \int_{0}^{\pi} \sin \theta \cos \theta S_{11}(\theta) \mathrm{d} \theta$.

In many lidar applications, a quantity called lidar ratio, $R$, is used. The lidar ratio is the ratio of the extinction to the backscattering cross-section:

$R=\frac{C_{\mathrm{ext}}}{C_{\mathrm{back}}}=\frac{k^{2} C_{\mathrm{ext}}}{S_{11}\left(180^{\circ}\right)}$,

where $S_{11}\left(180^{\circ}\right)$ is the value of $S_{11}$ at $\theta=180^{\circ}$, that is, the intensity at the exact backscattering direction.

Linear depolarization ratio, $\delta_{\mathrm{L}}$, is another quantity typically used in lidar applications. One reason is that the scattering matrix element $S_{22}$ is usually very sensitive to the shape of the particle, and therefore the linear depolarization ratio can be used to extract information about the particle shape, or at least to detect the presence of non-spherical particles. Linear depolarization ratio is defined as

$\delta_{\mathrm{L}}=\frac{S_{11}\left(180^{\circ}\right)-S_{22}\left(180^{\circ}\right)}{S_{11}\left(180^{\circ}\right)+S_{22}\left(180^{\circ}\right)}$.

As an example, single spherical, isotropic particles have $S_{11}=S_{22}$, which means that the linear depolarization ratio would be exactly zero.

\section{Light scattering simulations}

We performed the light scattering simulations of the generated inhomogeneous and homogeneous particles with a discrete dipole approximation (DDA) (Purcell and Pennypacker, 1973) light scattering software ADDA 1.2 MPI (Yurkin and Hoekstra, 2011). DDA is a flexible method for simulating light scattering by irregularly shaped particles, discretized into a regular lattice of individual dipoles. DDA is also capable of handling arbitrary collections of different materials: in an extreme case every lattice element can have a different refractive index. Therefore, DDA is extremely well suited for a detailed study of internal structures, such as that performed in this paper.

DDA is generally accurate as long as the target dipole resolution is sufficient. In this work, the target shapes for all size parameters were composed of roughly two hundred and twenty thousand dipoles. The value $y=|m| k l$, where $m$ is the refractive index, $k$ is the wavenumber and $l$ is the dipole size, is typically used to evaluate the applicability of the DDA method. The largest $y$ value for the particles in this study was approximately 0.7 , which is below the commonly cited DDA accuracy limit of $y \leq 1$ (Zubko et al., 2010).

Test simulations with higher dipole resolution for our inhomogeneous targets showed that $y=0.7$ already yields relative errors of several percent in differential scattering quantities. The use of higher dipole resolution at size parameters roughly $x \geq 16$ would therefore be beneficial, albeit very costly in terms of CPU time consumption. Regarding the size-distribution averaged results, on the other hand, where these largest sizes have only marginal weight, the relative errors are only about one percent. Our choice of dipole resolution is thus deemed quite sufficient.

We used a three-particle ensemble for results, and each internal structure case of each of these three particles was simulated with size parameters $\{0.5,1,2,3, \ldots, 20\}$. Furthermore, scattering by each inhomogeneous and homogeneous particle was averaged over 8192 random orientations for each size parameters.

The simulations were run on the Finnish Meteorological Institute Cray XC30 supercomputer Voima. In the calculations we used 64 computer cores per simulation, and 10 concurrent simulations were run in parallel. With this setup, the total amount of CPU time used was approximately $110000 \mathrm{~h}$ 
for three distinct versions of the five cases and their EMA versions.

\section{Particle generation model and scattering simulations}

\subsection{Generating the particle geometry}

Our method for generating the particle models involves multiple stages that can be run separately if needed. The overview of each main stage is given below. The algorithm assumes that the particle can be represented by a regular three-dimensional lattice of individual volume elements, or dipoles. With a good enough dipole resolution, the representation can replicate most large-scale structures of real dust particles with a sufficient accuracy. This representation also allows us to trivially convert the algorithm output to a DDA format for light scattering simulations. The generation process is summarized in Fig. 2.

\section{Stage 1: tessellation}

To generate computational models of realistic particles with internal structure, we employ an algorithm with threedimensional Voronoi tessellation at its core (Aurenhammer, 1991). The underlying idea is that the tessellation cells are roughly analog to the mineral grains within real (agglomerate) particles. While this is only an approximation, with the right mean size and shape of the grain, the method can be plausible. Voronoi tessellation has been used for irregular dust particle shape generation by e.g. Ishimoto et al. (2010), but our novel approach is to use the tessellation for generating internal particle structures instead of just the overall shape.

The generation of model shapes begins with an enclosed, discretized space composed of empty volume elements. The first step of the algorithm is to randomly place a given number of points within this volume. These act as seeds for the next step, which is to go through every element in the volume and to find the seed closest to it, as measured by a weighted distance, described below. The set of elements for which a given seed is closest forms a cell of that seed. Each element within a cell has the same composition as the seed, but different seeds can have different compositions.

The formula for weighted distance is:

$d_{w}=\sqrt{w_{x} \Delta x^{2}+w_{y} \Delta y^{2}+w_{z} \Delta z^{2}}$,

where $\Delta \cdot$ correspond to the distances along different axes, and $w$. correspond to the weight specific to different axes. In this formalism, a lower weight for an axis causes the grains to be elongated along that axis. The above equation is easily generalizable to have axis directions corresponding to arbitrary vectors, but in this case we have limited the directions to the major axes of the dipole lattice, hence the simplified form above. In this work we used $w_{x}=w_{y}=1, w_{z}=2 / 3$, to produce slightly elongated cells.

After the whole volume has been divided into cells, the volume is culled to extract a model particle from it. Here we have used an ellipsoid with the same axis proportions as the ellipsoidal grain axis proportions as the culling shape. The generated particles have aspect ratios close to 1.5. The culling is done in such a way that each cell with at least one element outside of the culling shape is removed from the volume, and the remaining cells form the particle. In this work we used 800 seeds within the original volume, yielding mean cell size of roughly 1200 elements, which translates to roughly $0.5 \%$ of the final culled particle volume.

\section{Stage 2: cell separation}

The next step is to separate the cells from each other. This is accomplished by finding the geometrical center of the particle, and forming unit vectors that point from it to the seeds of each cell. The cells are then moved to the direction specified by their corresponding vectors by a user-specified distance, and the final locations are discretized by rounding the cell element to the nearest integer. Therefore, the cells do not change sizes or shapes, but are separated from each other. This step is to allow separating individual cells or crystals from each other, which is often the case also with real dust particles and thus yields more realistic model shapes. The displacement length used in this work was 5, where 1 is the size of one lattice element, or a dipole in the DDA targets.

\section{Stage 3: concave hull}

The cell separation creates gaps between the cells, and the next step is to fill these gaps, and to soften sharp edges and other roughness characteristics around the particle. This is accomplished with a method called concave hull (Lindqvist et al., 2009). The concave hull method works by testing each element of the original volume in the following way: if a sphere with a constant radius $r$, a so-called generating sphere, centered at the element, does not overlap with any non-empty element, each element within the sphere is flagged. Otherwise, nothing is done. After all the elements have been checked with the generating sphere, all previously empty elements that have not been flagged are assigned to consist of a filling material with user-specified composition. The $r$ used in this work was three element lengths, which was enough to fill the inner seams caused by cell separation, and to partially fill some deep "valleys" at the surface.

\section{Stage 4: coating}

The fifth step is to coat the particles. The method for coating is simple: a layer of coating is added by setting each empty element that is orthogonally adjacent to a non-empty element as non-empty, assigning these elements to be composed of a coating material. Multi-layer coatings are formed by using 
the method iteratively. We used a three-layer coating in this work for both the normal coating and the hematite-rich coating cases.

\section{Stage 5: nodes (optional)}

There is an additional optional step, which can be used to add further internal structures in the form of inclusions. To generate inclusions in the original shape we insert nodes inside the particle. Nodes are generated simply by finding a random element in the particle, and growing a sphere of a given radius around the element, replacing parts of any existing cell with the node cell.

Here we have used nodes to generate both hematite inclusions and internal pores. The approach allows us to add these features into generated model particles without introducing any other changes in their shapes. For both the hematite nodes and internal pores, we generated 20 nodes with a radius of eight element lengths, which made the nodes comparable in size to cells.

\subsection{Assigning the mineral composition}

The above section contains the technical description of the shape-generating algorithm. The model is given physical relevance by introducing materials. Henceforth, each cell is stochastically assigned a material in such a way that the model particles would represent real dust particles composition as specified by the input parameters of the model. Mineral volume composition representative of Asian dust was derived from TEM and SEM data in Jeong and Achterberg (2014). Additionally, the concave hull filling cell, the coating cell, and the node cells from above are given a material corresponding to real-world materials.

The end product of this process is a list of volume elements that contain their position and refractive index. This is exactly what is needed for DDA simulations, hence making it straightforward to simulate scattering by these particles.

\subsection{Homogenization}

Once the particles with internal structure have been generated with the algorithm described above, we will generate their homogenized versions. This is achieved with a simple effective-medium approximation (EMA). We calculate the effective refractive index $m_{\text {eff }}$ of the homogenized particle as a volume-weighted average of the refractive indices of the constituent elements: (Chýlek et al., 2000)

$m_{\mathrm{eff}}=\sum_{i} f_{i} m_{i}$

where $f_{i}$ is the volume fraction of the $i$ th material, and $m_{i}$ is the refractive index of the $i$ th material.

Different mixing rules are known to perform differently depending on the particle type (Lesins et al., 2002). To ascertain that our results are not critically dependent on the choice of the mixing rule, we compared the performance of five different mixing rules for two different particles having the same shapes as our particles but being composed of only two constituent minerals: a clay particle with $15 \%$ of hematite as inclusions; and another clay particle with thin hematite-rich coating, where the hematitecontaining material volume fraction was $18 \%$. The mixing rules tested were the Maxwell Garnett, inverse Maxwell Garnett, Bruggeman, volume-weighted average refractive index (Eq. 7) and volume-weighted average permittivity. Scattering simulations were conducted with effective refractive indices produced by these rules, and compared with simulations where the inhomogeneity is explicitly accounted for. Differences in the obtained scattering matrix elements were then used to quantify the performance of the EMA in question.

For the first test particle, there was a very large variability between different EMA's, with the average refractive index and the inverse Maxwell Garnett EMA's performing the best. For the second test particle, all of the mixing rules performed decently at small sizes, but poorly at large sizes, and very similarly to each other at all sizes. Therefore, our conclusions is that out of the five mixing rules tested, not one performed better than the one selected here, and therefore the one selected here is appropriate for more detailed comparisons.

\section{Results}

\subsection{Particle generation results and the model correspondence with real dust particles}

In total, we studied five distinct internal structure cases. The five cases are:

- Case 1: an inhomogeneous particle composed of dielectrically similar minerals (no strong contrasts in the refractive index)

- Case 2: Case 1 particle with $15 \%$ hematite added as spherical inclusions (replacing the original material)

- Case 3: Case 1 particle with $17 \%$ air added as spherical internal pores (replacing the original material)

- Case 4: Case 1 particle with both hematite (15\%) and pores $(17 \%)$ added

- Case 5: Case 1 particle with the original coating material replaced by a hematite-rich material

For each case, the particle resembles the "baseline" Case 1 in other respects than the added features. Therefore, for instance, apart from the added hematite, the mineral volume fractions in Case 2 resemble those in Case 1. While they are not identical due to the stochastic nature of inclusion locations, they are close enough to plausibly assume that the 
Table 1. The mineral contents for each of the inhomogeneous cases. CM refers to clay mixture, Empty refers to internal pores, and HRCM refers to hematite-rich clay mixture. Volume fraction (VF) columns 1-5 correspond to Cases $1-5$. Percentages may not sum to $100 \%$ due to rounding in the displayed values. EMA $m$ values in the bottom part of the table show the homogenized refractive indices of each case.

\begin{tabular}{|c|c|c|c|c|c|c|c|}
\hline Mineral & \multicolumn{2}{|c|}{ Refractive index } & VF1 (\%) & VF2 (\%) & VF3 (\%) & VF4 (\%) & VF $5(\%)$ \\
\hline $\mathrm{CM}$ & & 1.55 & 31.19 & 39.05 & 36.42 & 21.78 & 13.26 \\
\hline Illite & & 1.57 & 21.06 & 14.88 & 14.88 & 14.88 & 21.06 \\
\hline Quartz & & 1.55 & 13.31 & 9.00 & 9.00 & 9.00 & 13.31 \\
\hline Smectite & & 1.52 & 12.77 & 7.96 & 7.96 & 7.96 & 12.77 \\
\hline Plagioclase & & 1.53 & 8.93 & 6.17 & 6.17 & 6.17 & 8.93 \\
\hline Calcite & & 1.60 & 3.85 & 2.37 & 2.37 & 2.37 & 3.85 \\
\hline Gypsum & & 1.52 & 2.78 & 1.66 & 1.66 & 1.66 & 2.78 \\
\hline Chlorite & & 1.58 & 2.19 & 1.59 & 1.59 & 1.59 & 2.19 \\
\hline K-eldspar & & 1.52 & 1.89 & 1.34 & 1.34 & 1.34 & 1.89 \\
\hline Kaolinite & & 1.56 & 1.86 & 1.16 & 1.16 & 1.16 & 1.86 \\
\hline Amphibole & & 1.62 & 0.18 & 0.16 & 0.16 & 0.16 & 0.18 \\
\hline Hematite & $3.09+\mathrm{i}$ & .0925 & 0.00 & 14.65 & 0.00 & 14.65 & 0.00 \\
\hline Empty & & 1.00 & 0.00 & 0.00 & 17.27 & 17.27 & 0.00 \\
\hline \multirow[t]{2}{*}{ HRCM } & $1.82+\mathrm{i}$ & 0139 & 0.00 & 0.00 & 0.00 & 0.00 & 17.93 \\
\hline & Case 1 & & & Case 3 & Case 4 & & \\
\hline EMA $m$ & 1.55 & 1.78 & 0.0135 & 1.46 & $68+i 0.0135$ & $1.60+\mathrm{i}$ & .0025 \\
\hline
\end{tabular}

changes seen are not caused by the differing non-hematite mineral content but instead are caused by hematite. In fact, in the text below we compare the other cases to Case 1 for specifically this reason.

For all of these cases, we use a three-particle ensemble for all of the results. We decided to use an ensemble to average out oscillations by single particles, and to see if the effects of different internal structures are consistent across all three different generated particles. Therefore, the particle generator is run three times with the same input parameters, the DDA simulations are run for all three versions of the five cases, and for each case we calculate the average of the three results. Because the generator is stochastic in nature, the three individual particles differ from each other despite having identical input parameters; however, all of the results are qualitatively similar for each individual particle in such a way that inter-particle variability is smaller than the difference between inhomogeneous and homogenized cases, or between inhomogeneous case and the baseline, for all scattering matrix elements and at most scattering angles.

Table 1 shows the ensemble-averaged mineral composition of the five different internal structure cases we have used here, as well as the EMA refractive indices for each case. The refractive indices for the minerals at $\lambda=550 \mathrm{~nm}$ are retrieved from MinDat database (http://www.mindat.org, accessed 21 May 2015). Empty is used to denote internal pores, CM to denote clay mixture, and HRCM to denote hematite-rich clay mixture. CM refractive index is calculated with EMA from the mineral composition of the first stage (tessellation) of the Case 1 particle, and HRCM refractive index is calculated likewise from the first stage of the Case 1 particle, but with $15 \%$ hematite content added. CM is used as the filling material, and as the coating material of all cases apart from Case 5. HRCM is used as the coating material of Case 4.

Due to the very large variability of the types and structures of real-world dust particles and the lack of information of the three-dimensional structures of the particles, quantitative validation of the generated shapes is challenging. Instead, we can compare the particle compositions to those of real particles, and compare the cross-sections visually. Looking at Fig. 3a, which depicts one example cross-section of Case 1 particle, we see that the grains are oriented and shaped in a somewhat random way. Figure 3 b shows the same particle, but with a three-dimensional rendering with a part of the particle cut off to show some of the internal structure. The grains of different materials seem to be scattered relatively randomly through the particle. Additionally, the characteristic sizes of the grains are generally $0.5-1 \mu \mathrm{m}$ with the largest $r$ used in this study, $1.75 \mu \mathrm{m}$. Comparing these parameters to the electron microscopy images by Jeong and Nousiainen (2014), especially the rock fragment dust particle (Fig. 13 in the reference), we see clear similarities. Although the model particle grains are slightly less irregular than some grains in the real particles, we see that the overall shapes, sizes and orientations are similar. However, the model particle grains are proportionally larger than those in the real rock fragment dust particle, with a radii of roughly $1 / 6$ of the particle radius instead of roughly $1 / 14$ of the particle radius that the rock fragment dust particle has. Still, since the model particles themselves are smaller than the particles shown in Jeong and Nousiainen (2014), the absolute sizes of the grains end up being 
(a)

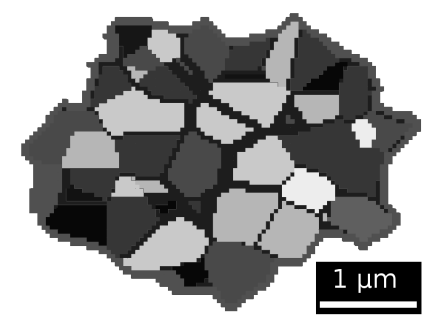

(c)

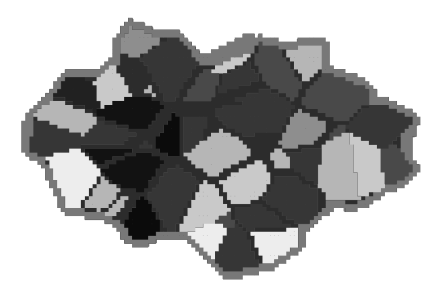

(b)

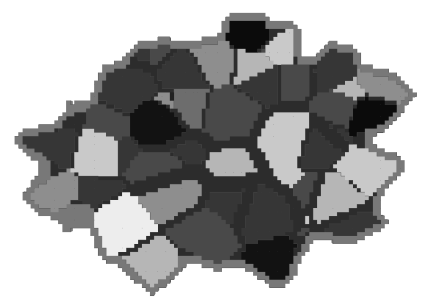

(d)

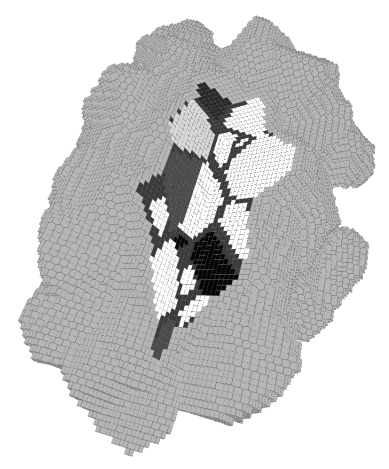

Figure 3. Example cross-sections of Case 1 versions of all three of the ensemble constituent particles (Panels a-c), and a threedimensional rendering (Panel d) of the particle in Panel (a), with a part of the particle cut out to reveal the inner structure. Different shades of gray correspond to different minerals. The scale bar in Panel (a) shows the approximate size of the features at the largest particle $r$ used in this study, $1.75 \mu \mathrm{m}$.

close to each other. One thing to note is that the coating thickness of the real dust particles seems to be roughly $0.5 \mu \mathrm{m}$, instead of roughly $0.1 \mu \mathrm{m}$ of the model particles. Regardless, as we show below, even this very thin coating causes clear effects when it contains hematite, and the effects would likely be only larger if the coating layer were thicker. Overall, although there are differences in details, we believe that our model particles are useful and sufficiently similar to observed real characteristics to serve as proxies in the sensitivity studies conducted here. Additionally, it could be argued that the correspondence of the particles does not matter significantly as long as they are not extremely unrealistic, such as very thin rods or spheres, as long as the internal features are of the correct size scale.

In addition to the grains and the coating, the model particles contain inclusions and pores, as described above. The nodes are generated to be comparable to grains in size, that is, diameters of $0.5-1 \mu \mathrm{m}$ at the largest size parameters studied (not shown). While internal pore sizes and shapes vary greatly in real dust particles, at least the iron-oxide-rich dust particle (Fig. 12 in Jeong and Nousiainen, 2014) shows pores with characteristic lengths of roughly $1 \mu \mathrm{m}$.

\subsection{Results of the light-scattering simulations}

Here we show the effect of taking inhomogeneity into account in light scattering simulations for several different internal structure scenarios. The scattering matrix elements will be analyzed as a function of the scattering angle after integrating the values over a size distribution. As the size distribution we use a lognormal distribution with the geometric mean radius $r_{\mathrm{g}}=0.4$ and the geometric standard deviation $\sigma_{\mathrm{g}}=2$, resulting in $r_{\mathrm{eff}}=0.82 \mu \mathrm{m}$. This distribution follows the one by Lindqvist et al. (2014), Kemppinen et al. (2015b), and is designed to provide reasonable contributions both from small and large size parameters, while also providing an effective radius reasonably close to those in real-world applications. Therefore, we kept the wavelength constant at $\lambda=550 \mathrm{~nm}$, while varying the particle $r$.

As the simulations are carried out separately for each particle size and only then averaged over the size distribution, we can easily estimate how a different choice of size distribution would impact the results. For example, had a wider size distribution been chosen, the results would have changed to some degree due to assigning a larger weight to larger particles compared to the current size distribution. Exact changes would depend on the inhomogeneity case and scattering matrix element in question, but based on the results for individual sizes, for example Case 5 EMA errors would have been increased. We speculate this is due to larger interaction between the radiation and the thin hematite-rich coating, causing the EMA to perform worse for large size parameters than for small size parameters.

It should be reiterated that the primary purpose here is not to study the single-scattering properties themselves, but how they differ when the internal structure is accounted for either explicitly or through an effective medium approximation. In particular, we are interested in establishing which types of internal structures have large effects on scattering. For each of the five cases, we show light scattering by the inhomogeneous particle (called IHG), light scattering by IHG Case 1 (called the baseline), as well as the homogeneous version of the particle (called EMA), for straightforward comparison of the effects of inhomogeneity. As a reminder, the baseline and the EMA versions of the particles are identical in size and shape to the corresponding IHG versions, and the only difference is in the local refractive indices within the lattice elements.

Below, we study each of the six independent scattering matrix elements separately. For each matrix element, we show all of the five cases, comparing the IHG version of the particle with the baseline and the EMA versions. Case 1 , the baseline, is not discussed separately along the other cases because EMA values for it are virtually identical to the IHG values for all of the scattering matrix elements. $S_{11}$ is shown as it is, using a logarithmic scale, and the other elements are shown as ratios $S$. / $S_{11}$, where $S$. is the element in 
question. Additionally, the ratios with $S_{12}$ and $S_{34}$ are shown as negatives instead, as per the usual convention.

Added contrast shows a clear effect on $S_{11}$, as seen in Fig. 4. In particular, added hematite nodes (Cases 2 and 4) and, to a lesser extent internal pores (Case 3), seem to decrease $S_{11}$ compared to the baseline Case 1 . The values at side-scattering and back-scattering angles are especially reduced. In addition to this reduction, the form of the scattering function is smoothed in these cases. It is notable that the smoother form of the scattering function resembles that of the real dust particles better than the Case 1 version (Muñoz et al., 2012), and tessellation particle simulations have been shown to overall compare reasonably well to laboratory scattering measurements (Ishimoto et al., 2010). Case 5, with the hematite-rich coating, does not differ noticeably from the baseline. For particles that differ from the baseline, Cases 2, 3 and 4, the performance of EMA for replicating the baseline varies. For the particle with added pores (Case 3), EMA seems to work reasonably well in replicating the IHG values, with only slight differences being seen at the sidescattering angles. However, for Cases 2 and 4, which include hematite inclusions, EMA values are clearly different from the true IHG values, in particular at the backscattering direction, where the EMA values can be up to $30 \%$ too large.

For $-S_{12} / S_{11}$ (Fig. 5), internal pores (Cases 3 and 4) seem to have the largest effects on scattering compared to the baseline, with the particle with only the hematite nodes (Case 2) also showing clear impact. For all of these cases, added internal structure increases $-S_{12} / S_{11}$ at most scattering angles. The particle with the hematite coating, Case 5, shows smaller difference to the baseline than the cases mentioned above, and the effect is of the opposite direction: the hematite-rich coating decreases $-S_{12} / S_{11}$ instead of increasing it. EMA seems to replicate the true scattering function of the IHG version relatively poorly for all of the Cases $2-5$. For Cases $2-4,-S_{12} / S_{11}$ for the EMA particle are lower than those of the corresponding IHG particles. However, for Case 5, EMA seems to smoothen out the angular dependency, and therefore the direction of the error varies depending on the scattering angle.

Figure 6 shows that hematite has a very significant effect on $S_{22} / S_{11}$ regardless of it being present as inclusions (Cases 2 and 4 ) or as a part of the coating material (Case 5). For Cases 2 and 4, the angle dependence of the scattering matrix values is overall smoother than that of the baseline case. Case 3 , with only the internal pores, also shows similar behavior to Cases 2 and 4, but with a much smaller magnitude. The particle with hematite coating (Case 5) also shows clear difference, but instead of the angular dependency being changed, the values are overall higher than the baseline. For $S_{22} / S_{11}$, the EMA versions of the particles generally do not replicate the true IHG values well. For Cases 2 and 4, the EMA values are slightly closer to the true values than the baseline, but the difference is still significant. For Case 3, EMA seems to, in fact, be further from the true values than the baseline.
Finally, for Case 5, the EMA values are virtually identical to the baseline values.

$S_{33} / S_{11}$ values (Fig. 7) for the IHG versions of the particles are generally lower than the baseline. The particle with internal pores, Case 3, is an exception, and for it the baseline and IHG are virtually identical. Interestingly, the difference between EMA values and the baseline is very small for all of the cases, in particular Cases 4 and 5, for which it is indistinguishable. For all of the cases, EMA results in larger than true values.

It is clear from Fig. 8 that the impact of internal structure on $-S_{34} / S_{11}$ varies significantly depending on the exact type of the structure. Hematite nodes in Cases 2 and 4 smoothen out the angular dependency greatly apart from forward-scattering angles, where the values are amplified. Because of this smoothing, the $-S_{34} / S_{11}$ values are overall smaller for the Case 2 and 4 IHG particles than those of the baseline. For the particle with internal pores, Case 3, the values are also smaller than the baseline, but the angular profile is not smoothed. For the hematite-coated Case 5, the IHG values are higher than those of the baseline, unlike for all of the other cases. Like with Case 3, the Case 5 angular dependency is not smoothed. Compared to the IHG and baseline values, the validity of EMA varies from case to case. For Cases 2 and 4, EMA works decently well, and although the values are not particularly close to those of IHG, at least they are closer than those of the baseline. However, for Cases 3 and 5, EMA values are more erroneous than even the baseline.

Lastly, the effects of the added forms of internal structure on $S_{44} / S_{11}$ are shown in Fig. 9, and are quite consistent for all of the cases studied. All of the IHG values are lower than those of the baseline, although for Case 3 the difference is clearly smaller than for the other cases. Again, the EMA versions of the particles do not replicate the $S_{44} / S_{11}$ values of the IHG versions closely. For Cases 2 and 3 the EMA values are slightly higher than the real values, while for Cases 4 and 5 the difference is higher. Interestingly, for Case 3 again, the scattering matrix element values for the EMA particle are further from the real values than the baseline.

As a practical consideration of identifying particle internal structures from measurements, we recommend polarization measurements. While producing an identification algorithm would require a very large amount of additional work, it seems that, for example, positive degree of linear polarization $\left(-S_{12} / S_{11}\right)$ values at scattering angles between 60 and $120^{\circ}$ correlate with refractive index contrasts inside the particles, whether that is from hematite nodes or internal cavities. However, it needs to be stressed that these observations apply only for single-scattering measurements; inferring particle internal structures from multiple-scattering polarization measurements will be much less straightforward. 
(a) Case 1

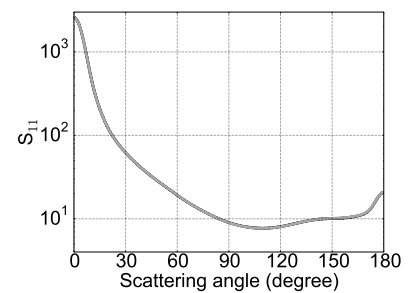

(d) Case 4

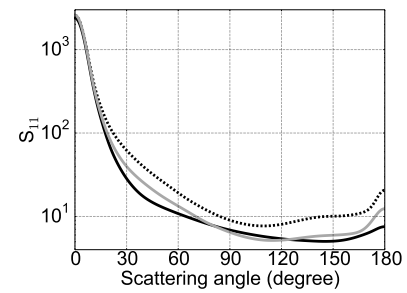

(b) Case 2

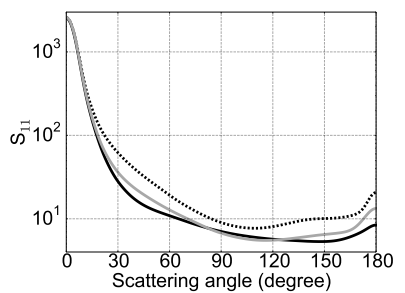

(e) Case 5

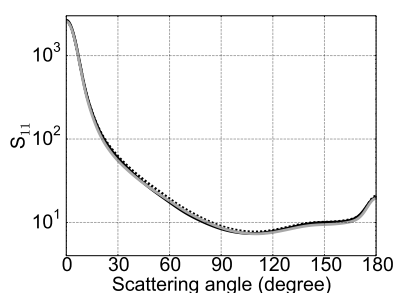

(c) Case 3
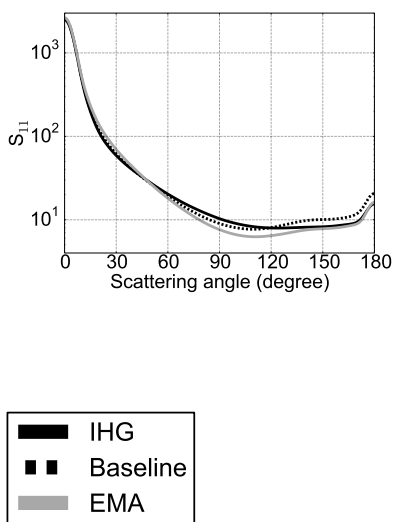

Figure 4. $S_{11}$ values for the inhomogeneous (IHG), the baseline, and the homogeneous (EMA) versions of all five of the internal structure cases as a function of the scattering angle.

(a) Case 1

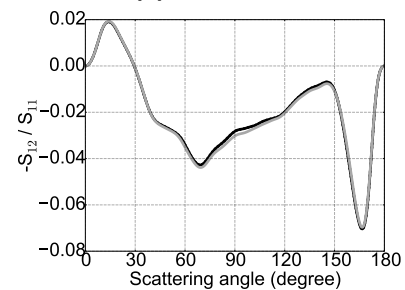

(d) Case 4

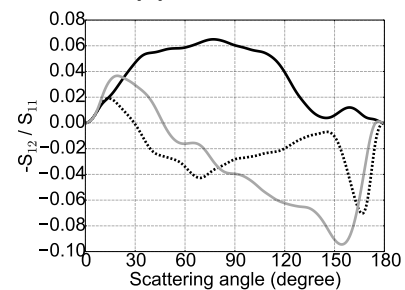

(b) Case 2

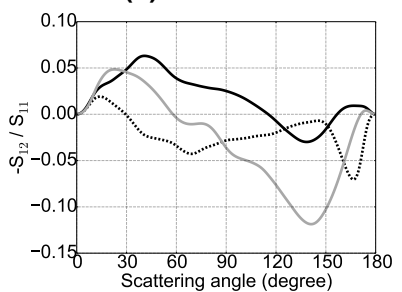

(e) Case 5

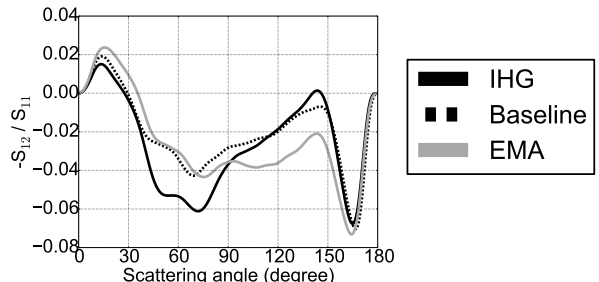

(c) Case 3

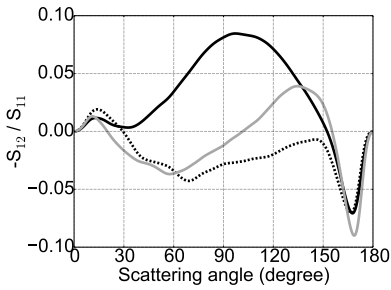

Scattering angle (degree)

Figure 5. $-S_{12} / S_{11}$ values for the inhomogeneous (IHG), the baseline, and the homogeneous (EMA) versions of all five of the internal structure cases as a function of the scattering angle.

\subsection{Scalar scattering quantities}

In addition to the effect of internal structures on the scattering matrix elements, we also explore the impacts of the same types of internal structures on four scalar quantities that are often used in climate or remote-sensing applications. These quantities, namely co-albedo, asymmetry parameter, linear depolarization ratio and lidar ratio, are shown below as a function of the particle size parameter. The format is similar to that used for the scattering matrix elements, where we show results separately for the IHG, the baseline, and the EMA particles, and compare them to see how added internal structures affect the values. In addition to the size- parameter-dependent figures, we also show the values of the size-distribution-averaged results in Table 2 for each case. In addition to the values themselves, the table also shows the difference, and the relative difference, that is, the difference as a percentage to the IHG value.

Single-scattering co-albedo for the original (IHG), Case 1 (baseline), and homogenized (EMA) versions of the threeparticle ensembles of the five internal structure cases are shown in Fig. 10. Neither Case 1 nor Case 3 contain any absorbing materials, and therefore their co-albedo are uniformly zero, and do not require any further discussion. Case 2 and Case 4 behave similarly to each other. For them, the co-albedo increases as the particle size increases due to the 
(a) Case 1

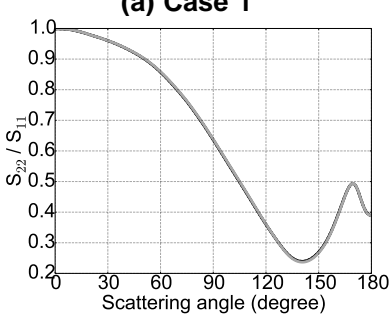

(d) Case 4

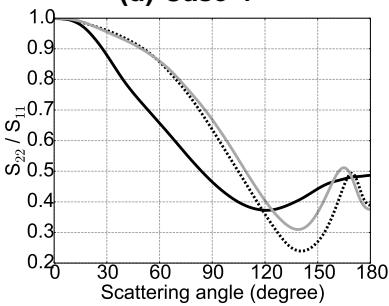

(b) Case 2

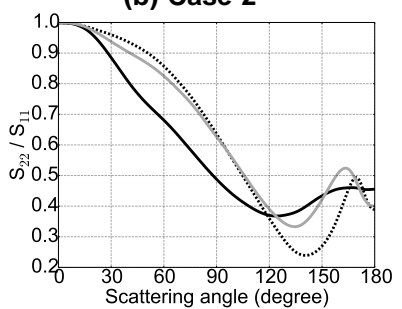

(e) Case 5

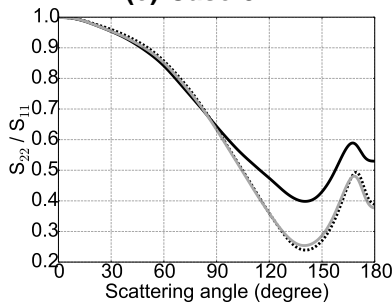

(c) Case 3

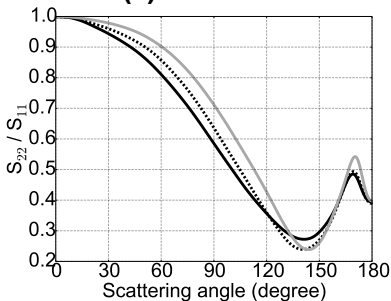

IIHG

- Baseline EMA

Figure 6. $S_{22} / S_{11}$ values for the inhomogeneous (IHG), the baseline, and the homogeneous (EMA) versions of all five of the internal structure cases as a function of the scattering angle.

(a) Case 1

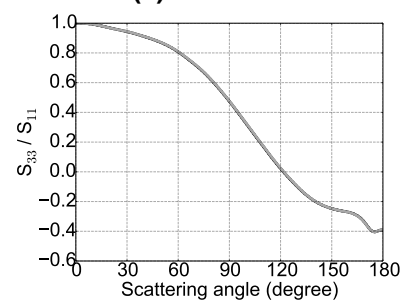

(d) Case 4

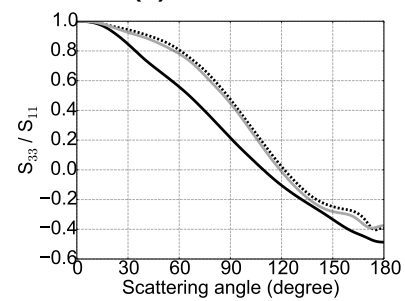

(b) Case 2

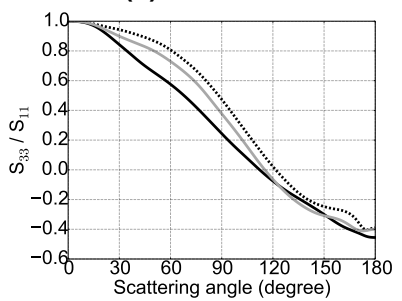

(e) Case 5

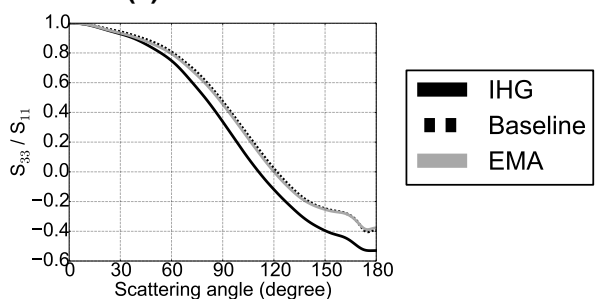

(c) Case 3

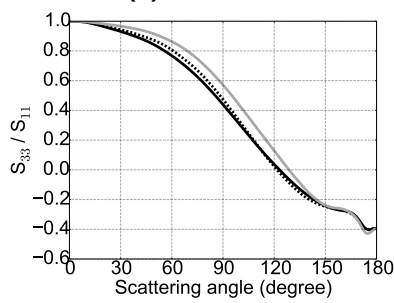

Scattering angle (degree)

Figure 7. $S_{33} / S_{11}$ values for the inhomogeneous (IHG), the baseline, and the homogeneous (EMA) versions of all five of the internal structure cases as a function of the scattering angle.

hematite inclusions approaching the wavelength size. For sizes where the inclusions are larger than the wavelength of the incoming radiation, roughly size parameter 10 and larger, the co-albedo stabilizes to an almost constant value. For these cases, EMA underestimates co-albedo of the IHG version by $15-50 \%$ between size parameters roughly 5 and 15 . At small sizes, the inclusions are much smaller than the wavelength, and therefore the EMA and the IHG values are close to each other. At the largest sizes, it seems like EMA co-albedo is approaching the values of the IHG particle and becoming even larger. It would be interesting to see if at very large size parameters this development continues and EMA ends up overestimating co-albedo significantly. For Case 5, the co-albedo increases almost linearly as the size parameter increases, when the coating starts to interact with light more strongly. With increased coating thickness or even larger particle sizes, it is reasonable to assume that the co-albedo would increase even further. Case 5 also shows a small, but consistent, difference in co-albedo between the IHG and the EMA particles, with EMA being higher.

Asymmetry parameter for the original and homogenized versions of the five internal structure cases is shown in Fig. 11. For the particles with hematite nodes, Cases 2 and 4 , the asymmetry parameter is higher than that of the baseline by roughly $20 \%$ at most size parameters. For the particles with only the pores added (Case 3 ) and the particles with the 
(a) Case 1

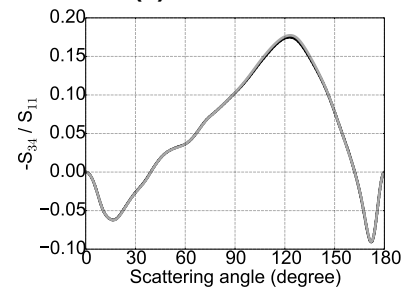

(d) Case 4

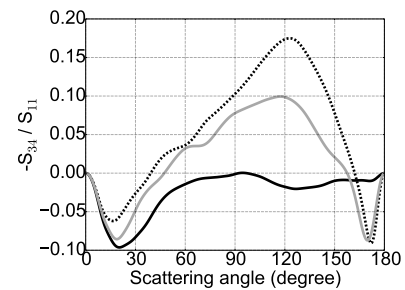

(b) Case 2

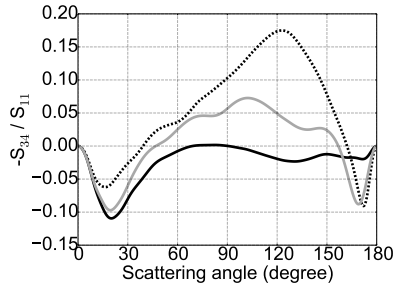

(e) Case 5

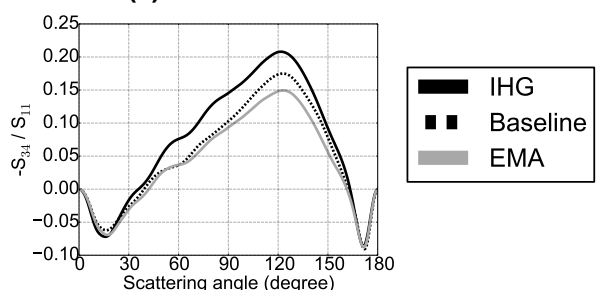

(c) Case 3

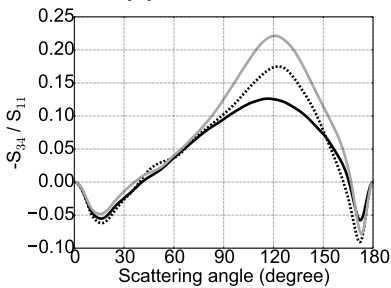

Figure 8. $-S_{34} / S_{11}$ values for the inhomogeneous (IHG) and the homogeneous (EMA) versions of all five of the internal structure cases as a function of the scattering angle.

(a) Case 1

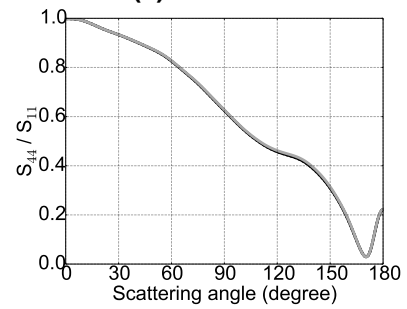

(d) Case 4

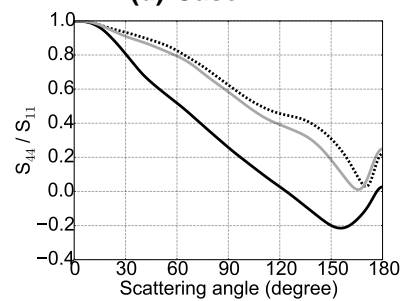

(b) Case 2

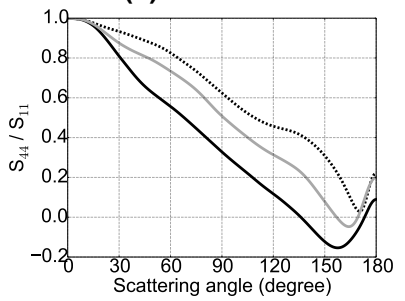

(e) Case 5

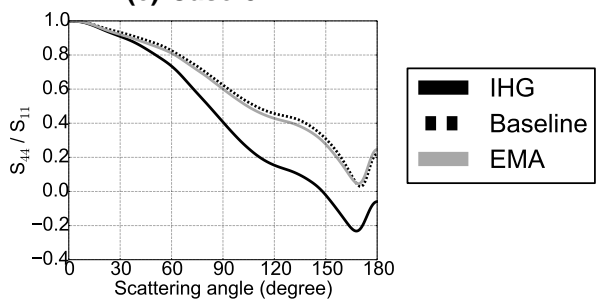

(c) Case 3

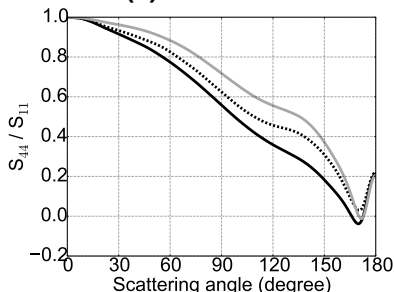

Scattering angle (degree)

Figure 9. $S_{44} / S_{11}$ values for the inhomogeneous (IHG), the baseline, and the homogeneous (EMA) versions of all five of the internal structure cases as a function of the scattering angle.

hematite coating (Case 5), the asymmetry parameter is virtually identical to the baseline. For all of the particles shown here, EMA performs reasonably well for the asymmetry parameters, following the IHG values closely.

The linear depolarization ratio for the original and homogenized versions of the five internal structure cases are shown in Fig. 12. Added hematite seems to have a very significant increase on linear depolarization ratio, regardless of whether it is present as inclusions (Cases 2 and 4) or as part of the coating material (Case 5). The effect of hematite is to decrease linear depolarization ratio by up to $40 \%$ at large size parameters, and coating seems to have a stronger impact than inclusions. Internal pores have a smaller effect, and increase the linear depolarization rate by roughly $10 \%$. For Cases 2 and 4, EMA is closer to the true IHG values than the baseline, but the difference is still notable. However, for Cases 3 and 5 , EMA performs badly and has values very close to those of the baseline.

Lidar ratio for the original and homogenized versions of the five internal structure cases are shown in Fig. 13. Hematite nodes (Cases 2 and 4) seem to affect the lidar ratio greatly, whereas internal pores or hematite coating (Cases 3 and 5) do not. At large size parameters, the presence of hematite nodes roughly triples the lidar ratio from the baseline values, and even at smaller size parameters, the difference is notable. For Cases 2 and 4, EMA results are typi- 
(a) Case 1

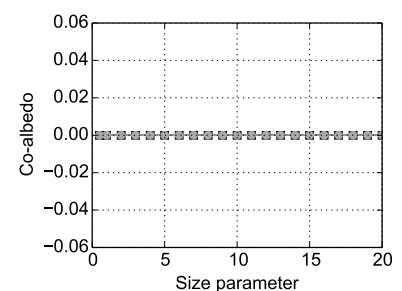

(d) Case 4

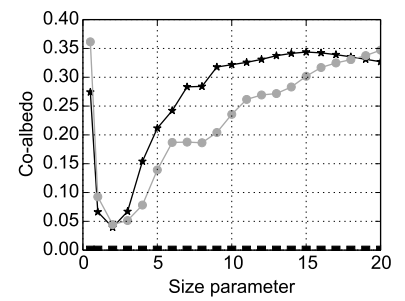

(b) Case 2

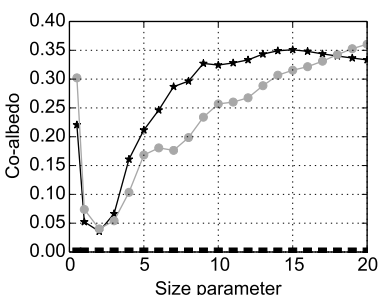

(e) Case 5

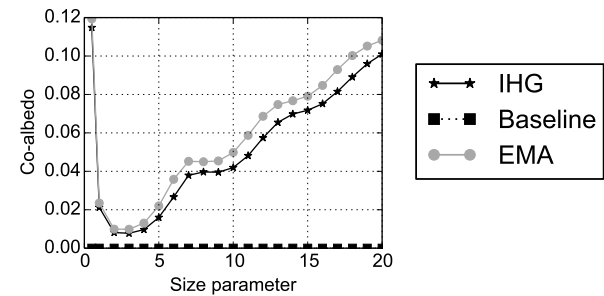

(c) Case 3
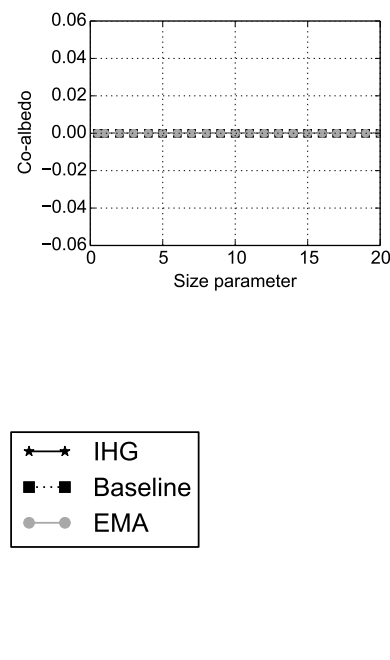

Figure 10. Co-albedo for the inhomogeneous (IHG), the baseline, and the homogeneous (EMA) versions of all five particle cases as a function of the size parameter of the particle.

Table 2. Scalar scattering quantities for the size distribution averaged three-particle ensembles. Inhomogeneous (IHG), Case 1 (baseline) and homogeneous (EMA) values are shown separately, as well as their differences as percentages of the IHG value.

\begin{tabular}{|c|c|c|c|c|c|}
\hline Case & IHG & Baseline & EMA & (IHG - Baseline) / IHG (\%) & $(\mathrm{IHG}$ - EMA) / IHG (\%) \\
\hline \multicolumn{6}{|c|}{ Co-albedo } \\
\hline Case 1 & 0.00 & 0.00 & 0.00 & $\mathrm{n} / \mathrm{a}$ & $\mathrm{n} / \mathrm{a}$ \\
\hline Case 2 & 0.18 & 0.00 & 0.14 & 100.00 & 22.22 \\
\hline Case 3 & 0.00 & 0.00 & 0.00 & $\mathrm{n} / \mathrm{a}$ & $\mathrm{n} / \mathrm{a}$ \\
\hline Case 4 & 0.18 & 0.00 & 0.13 & 100.00 & 27.78 \\
\hline Case 5 & 0.02 & 0.00 & 0.03 & 100.00 & -50.00 \\
\hline \multicolumn{6}{|c|}{ Asymmetry parameter } \\
\hline Case 1 & 0.62 & 0.62 & 0.62 & 0.00 & 0.04 \\
\hline Case 2 & 0.57 & 0.62 & 0.58 & -8.46 & -0.66 \\
\hline Case 3 & 0.64 & 0.62 & 0.68 & 2.89 & -5.88 \\
\hline Case 4 & 0.59 & 0.62 & 0.61 & -6.19 & -4.09 \\
\hline Case 5 & 0.62 & 0.62 & 0.61 & -0.25 & 2.41 \\
\hline \multicolumn{6}{|c|}{ Linear depolarization ratio } \\
\hline Case 1 & 0.31 & 0.31 & 0.31 & 0.00 & 0.19 \\
\hline Case 2 & 0.35 & 0.31 & 0.35 & 13.30 & -0.04 \\
\hline Case 3 & 0.25 & 0.31 & 0.25 & -24.33 & -0.58 \\
\hline Case 4 & 0.32 & 0.31 & 0.35 & 3.54 & -10.60 \\
\hline Case 5 & 0.26 & 0.31 & 0.33 & -19.88 & -29.21 \\
\hline \multicolumn{6}{|c|}{ Lidar ratio } \\
\hline Case 1 & 45.51 & 45.51 & 45.59 & 0.00 & -0.17 \\
\hline Case 2 & 44.26 & 45.51 & 34.08 & -2.83 & 23.00 \\
\hline Case 3 & 57.09 & 45.51 & 66.28 & 20.28 & -16.10 \\
\hline Case 4 & 47.81 & 45.51 & 39.89 & 4.80 & 16.57 \\
\hline Case 5 & 40.58 & 45.51 & 39.83 & -12.14 & 1.85 \\
\hline
\end{tabular}


(a) Case 1

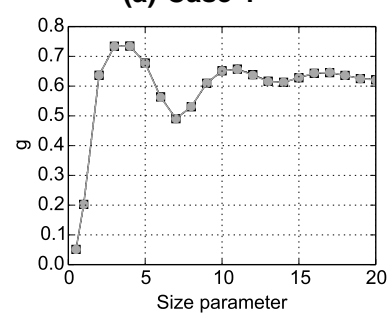

(d) Case 4

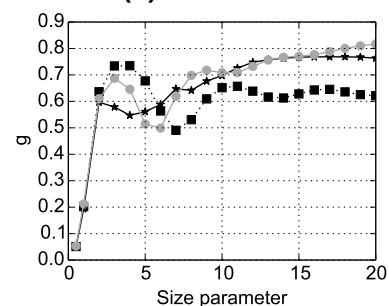

(b) Case 2

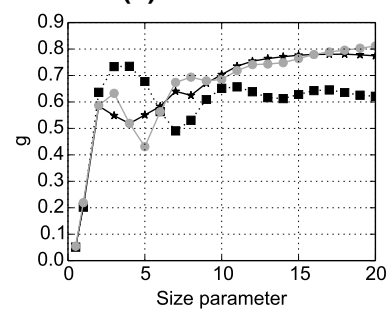

(e) Case 5

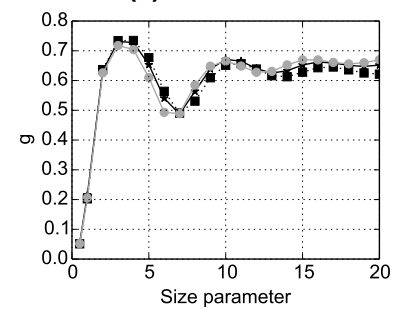

(c) Case 3

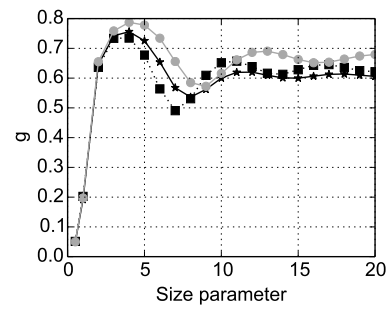

- Baseline

$\rightarrow$ EMA

Figure 11. Asymmetry parameter for the inhomogeneous (IHG), the baseline, and the homogeneous (EMA) versions of all five particle cases as a function of the size parameter of the particle.

(a) Case 1

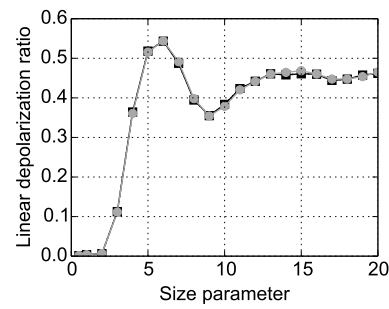

(d) Case 4

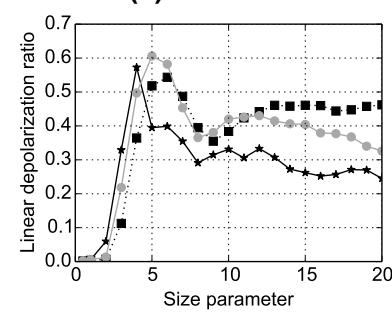

(b) Case 2

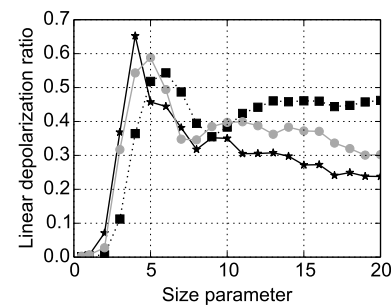

(e) Case 5

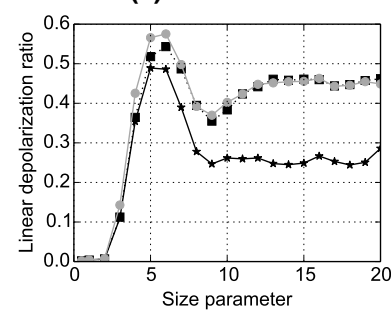

(c) Case 3

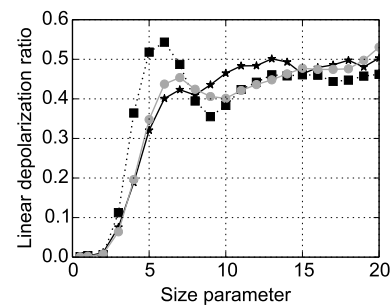

ઋ

-... Baseline

$\because$ EMA

Figure 12. Linear depolarization ratio for the inhomogeneous (IHG), the baseline, and the homogeneous (EMA) versions of all five particle cases as a function of the size parameter of the particle.

cally between those for the baseline and IHG, meaning that the EMA manages to partially account for the impact of the additional internal structure. Still, EMA results differ from those of IHG by $20-50 \%$. The direction of the difference between IHG and EMA depend on the exact size parameter. For Cases 3 and 5, EMA values are indistinguishable from the IHG values.

\section{Summary and conclusions}

In this work, we studied the effects of dust particle internal structure in a computational way based on real internal structures revealed by Jeong and Nousiainen (2014). First, we generated qualitatively realistic dust particles with various internal structures by using a sophisticated computational model. Second, homogeneous versions of these particles were generated with an effective-medium approximation. Third, light scattering simulations for both versions of the particles were run with a discrete dipole approximation program called ADDA. 
(a) Case 1

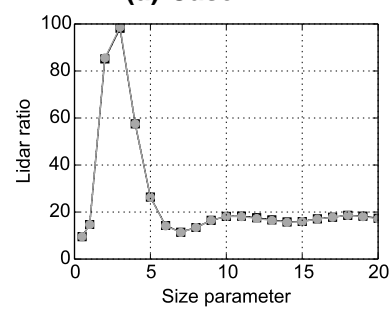

(d) Case 4

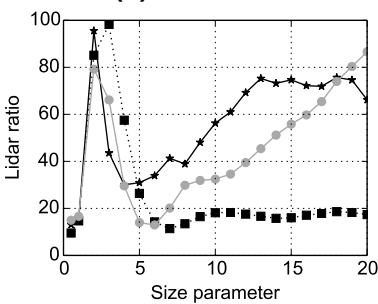

(b) Case 2

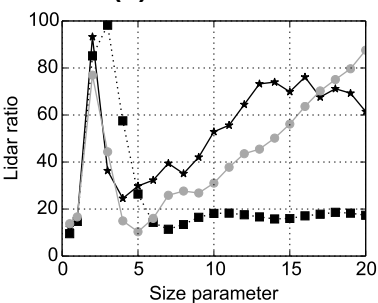

(e) Case 5

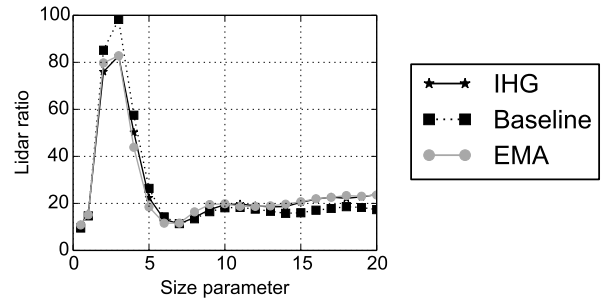

(c) Case 3

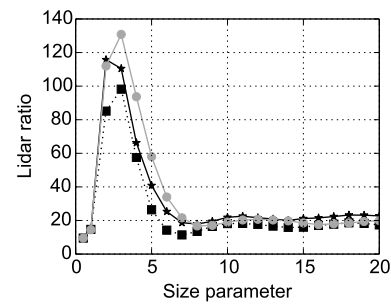

Figure 13. Lidar ratio for the inhomogeneous (IHG), the baseline, and the homogeneous (EMA) versions of all five particle cases as a function of the size parameter of the particle.

Five distinct internal structure cases were studied, and for each case we used a three-particle ensemble. First, we studied particles whose composite minerals had similar refractive indices. This was considered our baseline, to which the other cases were compared to. Second, we added hematite inclusions to the baseline particles. Third, we added internal pores to the baseline. Fourth, both hematite inclusions and internal pores were added to the baseline. Fifth, the coating material of the baseline was replaced with a hematite-containing material, but no nodes or pores were added.

These models of internal structures were selected by their common occurrence in the Asian dust particles on the basis of systematic TEM data provided in Jeong and Nousiainen (2014) Although many more cases are possible, we restricted the analysis to five cases to include major features of internal structures while keeping the computation load manageable. Natural dust particles contain two major mineral types of iron oxides: goethite and hematite. We selected hematite as a representative iron oxide in the structural model because its high refractive indices and effect on optical properties were treated previously (Sokolik and Toon, 1999; Lafon et al., 2006). Of course, iron oxide contents of dust particles vary in a wide range, thus $15 \%$ volume hematite observed in natural particle is likely a case of a rather high content of iron oxides. However, the purpose of the calculation here was to show clearly the effect of hematite on diverse optical properties.

For each of these cases, we studied light scattering by both the inhomogeneous and homogenized versions of the particles and compared them against the baseline. The results show that most types of internal structure have clear effects on light scattering, and that many of those effects are not properly accounted for by the effective-medium ap- proximation (EMA) that we used. Our findings are consistent with those of Kocifaj and Videen (2008) that the performance of EMA is inconsistent when the inhomogeneity is on macroscopic scale. Possible errors from the use of EMA's for such particles are thus hard to predict, and will depend on the quantity of interest. Overall, hematite inclusions turned out to be the most impactful of the forms of internal structure studied here, affecting all of the scattering matrix elements and scalar scattering quantities. Hematite-rich coating affects all of the scattering matrix elements apart from $S_{11}$, and for linear depolarization ratio has an even greater effect than hematite inclusions. Internal pores affect especially $-S_{12} / S_{11}$ very significantly, but also the other scattering matrix elements to a lesser degree; scalar scattering quantities considered, on the other hand, remain relatively close to the baseline. Additionally, in many cases, having both hematite nodes and internal pores present amplifies the effects compared to having only the hematite nodes, instead of dampening the effects in some way. Finally, having a particle composed of several different materials, but with similar refractive indices, is the exception. In our analysis such a particle could safely be treated as homogeneous, and even using a very simple mixing rule for the effective refractive index calculations provided accurate results.

All four of the scalar variables studied, the singlescattering co-albedo, asymmetry parameter, linear depolarization ratio and lidar ratio, were affected noticeably by some forms of internal structure. For co-albedo, adding hematite content increased the values significantly. For asymmetry parameter, hematite nodes, but not hematite-rich coating, increased the values clearly. For linear depolarization ratio, all forms of hematite lowered the values greatly. Finally, for lidar ratio, added hematite nodes increased the values two- or 
three-fold. Interestingly, we can compare the differences to those caused by adding surface roughness, as done by Kemppinen et al. (2015a). Overall, internal structures, especially hematite nodes, seem to have a greater effect on scattering matrix elements than modest surface roughness. However, the impact of surface roughness on scattering at the backscattering direction can be very notable, comparable to internal hematite. For example, increasing surface roughness has a similarly sized effect on linear depolarization ratio as that of adding hematite, but of the opposite direction.

Based on these results, it appears that the internal structure of real dust particles needs to be accounted for in singlescattering simulations to obtain accurate results. Not only is it common in real dust particles, it also has major effects on scattering matrix elements and many scalar scattering quantities. Furthermore, the form of the internal structure matters. For example, light scattering changes considerably depending on whether hematite is present as inclusions, or mixed in the coating material. Additionally, at least for the simple mixing rule tested here, a homogenized particle created with an effective-medium approximation is unable to well mimic scattering by the original inhomogeneous version of the particle in most cases. In fact, in some cases using an effectivemedium approximation causes results to be more wrong than ignoring the internal structure altogether. Therefore, if accuracy is desired in the results, accounting for internal structure should be done explicitly.

Following up on these results, there are several directions to consider. As a practical concern, one might try to find an EMA that works very well for some or all of the inhomogeneity types here. Additionally, replicating the scattering by the inhomogeneous particles by using detailed modeling results to fine-tune shape and composition ensembles of simple model shapes, such as ellipsoids, might lead to much better results in applications. However, as shown by Kemppinen et al. (2015b), such a fitting procedure is risky, and needs to be done with caution. Without either of the above options, the results are still hard to apply in practical applications, such as retrieval algorithms or climate models. On the positive side, the differences seen between inhomogeneity types may help in identifying dust particle types from remote measurements, especially polarization and lidar measurements. While this requires a great deal of work, there is clearly hope that such a method could be developed.

\section{The Supplement related to this article is available online at doi:10.5194/acp-15-12011-2015-supplement.}

Acknowledgements. The authors wish to thank Maxim Yurkin, Bastiaan van Diedenhoven and an anonymous referee for their helpful comments in improving the manuscript. This research has been funded by the Academy of Finland (grant 255718), the
Finnish Funding Agency for Technology and Innovation (Tekes; grant 3155/31/2009), the Magnus Ehrnrooth Foundation, and the National Research Foundation of Korea grant NRF-2011-0028597. Maxim Yurkin is acknowledged for making his ADDA code publicly available (https://code.google.com/p/a-dda/).

Edited by: J. Allan

\section{References}

Aurenhammer, F.: Voronoi diagrams a survey of a fundamental geometric data structure, ACM Comput. Surv., 23, 345-405, 1991.

Chou, C., Formenti, P., Maille, M., Ausset, P., Helas, G., Harrison, M., and Osborne, S.: Size distribution, shape, and composition of mineral dust aerosols collected during the African monsoon multidisciplinary analysis special observation period 0 : dust and biomass-burning experiment field campaign in Niger, January 2006, J. Geophys. Res.-Atmos., 113, D00C10, doi:10.1029/2008JD009897, 2008.

Chýlek, P., Videen, G., Geldart, D. J. W., Dobbie, J. S., and Tso, H. C. W.: Effective medium approximations for heterogeneous particles, in: Light Scattering by Nonspherical Particles, edited by: Mishchenko, M. I., Hovenier, J. W., and Travis, L. D., chap. 9, Academic Press, San Diego, USA, 273-308, 2000.

Durant, A., Harrison, S., Watson, I., and Balkanski, Y.: Sensitivity of direct radiative forcing by mineral dust to particle characteristics, Prog. Phys. Geog., 33, 80-102, 2009.

Forster, P., Ramaswamy, V., Artaxo, P., Berntsen, T., Betts, R., Fahey, D., Haywood, J., Lean, J., Lowe, D., Myhre, G., Nganga, J., Prinn, R., Raga, G., Schulz, M., and Dorland, R. V.: Changes in atmospheric constituents and in radiative forcing, in: Climate Change 2007: The Physical Science Basis, Contribution of Working Group I to the Fourth Assessment Report of the Intergovernmental Panel on Climate Change, edited by: Solomon, S., Qin, D., Manning, M., Chen, Z., Marquis, M., Averyt, K., Tignor, M., and Miller, H., chap. 2, Cambridge University Press, Cambridge, UK and New York, NY, USA, 130-234, 2007.

Haywood, J., Johnson, B., Osborne, S., Baran, A., Brooks, M., Milton, S., Mulcahy, J., Walters, D., Allan, R. P., Klaver, A., et al.: Motivation, rationale and key results from the GERBILS Saharan dust measurement campaign, Q. J. Roy. Meteorol. Soc., 137, 1106-1116, 2011a.

Haywood, J. M., Johnson, B. T., Osborne, S. R., Mulcahy, J., Brooks, M. E., Harrison, M. A. J., Milton, S. F., and Brindleye, H. E.: Observations and modelling of the solar and terrestrial radiative effects of Saharan dust: a radiative closure case-study over oceans during the GERBILS campaign, Q. J. Roy. Meteorol. Soc., 137, 1211-1226, doi:10.1002/qj.770, 2011 b.

Hovenier, J. and van der Mee, C.: Basic relationships for matrices describing scattering by small particles, in: Light Scattering by Nonspherical Particles, edited by: Mishchenko, M. I., Hovenier, J. W., and Travis, L. D., chap. 3, Academic Press, San Diego, USA, 61-85, 2000.

Ishimoto, H., Zaizen, Y., Uchiyama, A., Masuda, K., and Mano, Y.: Shape modeling of mineral dust particles for light-scattering calculations using the spatial Poisson-Voronoi tessellation, J. Quant. Spectrosc. Ra., 111, 2434-2443, 2010. 
Jeong, G. Y. and Achterberg, E. P.: Chemistry and mineralogy of clay minerals in Asian and Saharan dusts and the implications for iron supply to the oceans, Atmos. Chem. Phys., 14, 1241512428, doi:10.5194/acp-14-12415-2014, 2014.

Jeong, G. Y. and Nousiainen, T.: TEM analysis of the internal structures and mineralogy of Asian dust particles and the implications for optical modeling, Atmos. Chem. Phys., 14, 7233-7254, doi:10.5194/acp-14-7233-2014, 2014.

Jeong, G. Y., Kim, J. Y., Seo, J., Kim, G. M., Jin, H. C., and Chun, Y.: Long-range transport of giant particles in Asian dust identified by physical, mineralogical, and meteorological analysis, Atmos. Chem. Phys., 14, 505-521, doi:10.5194/acp-14-505-2014, 2014.

Kemppinen, O., Nousiainen, T., and Lindqvist, H.: The impact of surface roughness on scattering by realistically shaped wavelength-scale dust particles, J. Quant. Spectrosc. Ra., 150, 55-67, doi:10.1016/j.jqsrt.2014.05.024, 2015a.

Kemppinen, O., Nousiainen, T., Merikallio, S., and Räisänen, P.: Retrieving microphysical properties of dust-like particles using ellipsoids: the case of refractive index, Atmos. Chem. Phys., 15, 11117-11132, doi:10.5194/acp-15-11117-2015, 2015 b.

Kim, S.-W., Yoon, S.-C., and Kim, J.: Columnar Asian dust particle properties observed by sun/sky radiometers from 2000 to 2006 in Korea, Atmos. Environ., 42, 492-504, 2008.

Kocifaj, M. and Videen, G.: Optical behavior of composite carbonaceous aerosols: DDA and EMT approaches, J. Quant. Spectrosc. Ra., 109, 1404-1416, doi:10.1016/j.jqsrt.2007.11.007, 2008.

Lafon, S., Sokolik, I. N., Rajot, J. L., Caquineau, S., and Gaudichet, A.: Characterization of iron oxides in mineral dust aerosols: implications for light absorption, J. Geophys. Res.Atmos., 111, D21207, doi:10.1029/2005JD007016, 2006.

Lesins, G., Chylek, P., and Lohmann, U.: A study of internal and external mixing scenarios and its effect on aerosol optical properties and direct radiative forcing, J. Geophys. Res.-Atmos., 107, AAC 5-1-AAC 5-12, doi:10.1029/2001JD000973, 2002.

Lindqvist, H., Muinonen, K., and Nousiainen, T.: Light scattering by coated Gaussian and aggregate particles, J. Quant. Spectrosc. Ra., 110, 1398-1410, doi:10.1016/j.jqsrt.2009.01.015, 2009.

Lindqvist, H., Jokinen, O., Kandler, K., Scheuvens, D., and Nousiainen, T.: Single scattering by realistic, inhomogeneous mineral dust particles with stereogrammetric shapes, Atmos. Chem. Phys., 14, 143-157, doi:10.5194/acp-14-143-2014, 2014.

Muinonen, K., Nousiainen, T., Lindqvist, H., Muñoz, O., and Videen, G.: Light scattering by Gaussian particles with internal inclusions and roughened surfaces using ray optics, J. Quant. Spectrosc. Ra., 110, 1628-1639, doi:10.1016/j.jqsrt.2009.03.012, 2009.

Muñoz, O., Moreno, F., Guirado, D., Dabrowska, D., Volten, H., and Hovenier, J.: The Amsterdam-Granada light scattering database, J. Quant. Spectrosc. Ra., 113, 565-574, 2012.
Nousiainen, T. and Kandler, K.: Light scattering by atmospheric mineral dust particles, in: Light Scattering Reviews 9, edited by: Kokhanovsky, A. A., Springer Praxis Books, Springer, Berlin, Heidelberg, Germany, 3-52, doi:10.1007/978-3-642-37985-7, 2015.

Nousiainen, T., Muinonen, K., and Räisänen, P.: Scattering of light by large Saharan dust particles in a modified ray optics approximation, J. Geophys. Res., 108, 4025, doi:10.1029/2001JD001277, 2003.

Nousiainen, T., Kahnert, M., and Lindqvist, H.: Can particle shape information be retrieved from light-scattering observations using spheroidal model particles?, J. Quant. Spectrosc. Ra., 112, 2213 2225, doi:10.1016/j.jqsrt.2011.05.008, 2011a.

Nousiainen, T., Muñoz, O., Lindqvist, H., Mauno, P., and Videen, G.: Light scattering by large Saharan dust particles: comparison of modeling and experimental data for two samples, J. Quant. Spectrosc. Ra., 112, 420-433, doi:10.1016/j.jqsrt.2010.09.003, 2011 b.

Osborne, S., Baran, A., Johnson, B., Haywood, J., Hesse, E., and Newman, S.: Short-wave and long-wave radiative properties of Saharan dust aerosol, Q. J. Roy. Meteorol. Soc., 137, 1149-1167, 2011.

Purcell, E. M. and Pennypacker, C. R.: Scattering and absorption of light by nonspherical dielectric grains, Astrophys. J., 186, 705$714,1973$.

Sokolik, I. R. and Toon, O. B.: Incorporation of mineralogical composition into models of the radiative properties of mineral aerosol from UV to IR wavelengths, J. Geophys. Res., 104, 9423-9444, 1999.

Vilaplana, R., Moreno, F., and Molina, A.: Study of the sensitivity of size-averaged scattering matrix elements of nonspherical particles to changes in shape, porosity and refractive index, J. Quant. Spectrosc. Ra., 100, 415-428, doi:10.1016/j.jqsrt.2005.11.068, 2006.

Woodward, X., Kostinski, A., China, S., Mazzoleni, C., and Cantrell, W.: Characterization of dust particles' 3D shape and roughness with nanometer resolution, Aerosol Sci. Tech., 49, 229-238, 2015.

Yurkin, M. A. and Hoekstra, A. G.: The discrete-dipoleapproximation code ADDA: capabilities and known limitations, J. Quant. Spectrosc. Ra., 112, 2234-2247, 2011.

Zender, C. S., Bian, H., and Newman, D.: The mineral Dust Entrainment And Deposition (DEAD) model: description and 1990s dust climatology, J. Geophys. Res., 108, 4416, doi:10.1029/2002JD002775, 2003.

Zubko, E., Petrov, D., Grynko, Y., Shkuratov, Y., Okamoto, H., Muinonen, K., Nousiainen, T., Kimura, H., Yamamoto, T., and Videen, G.: Validity criteria of the discrete dipole approximation, Appl. Optics, 49, 1267-1279, 2010. 